\title{
Does 'Immortal DNA strand' exist in 'immortal' stem cells?
}

\author{
Linheng $\mathrm{Li}^{1}$ \\ ${ }^{1}$ Stowers Institute for Medical Research, Kansas City, MO 64110, USA. LIL@Stowers-Institute.org \\ Cell Research (2007) 17:834-835. doi: 10.1038/cr.2007.87; published online 15 October 2007
}

Stem cells function to generate differentiated cells, and, at the same time, are maintained as 'immortal' cells through self-renewal. Accumulated evidence indicates that adult stem cells may be the most common precursor for cancers in adult mammalian tissue [1], and this concept is gaining increasing support $[2,3]$. Meanwhile, this also raises the question of how stem cells, which have a much longer lifespan compared to other progenitor and mature cells, can avoid accumulating mutations from DNA replication errors, thereby reducing the risk of tumorigenesis. In 1975, J Cairns proposed an intriguing hypothesis from an evolutionary point of view to explain this phenomenon in organisms with long life spans. According to this 'immortal DNA strand' model, adult stem cells might be able to retain template (old) DNA strands in each asymmetric division to avoid accumulation of mutations during the process of DNA replication [4].

Although there is no molecular mechanism thus far to support this long-standing attractive model in vivo, asymmetric segregation of labeled DNA strands during stem cell division or in some mutant cell lines has been observed. Related to these observations, a technique termed longterm retention cell (LRC), has been used to determine the putative stem cell location in mammals. If a cell's DNA is incorporated with isotope-labeled substrate ( ${ }^{3} \mathrm{H}$-thymidine) or an analogue of thymidine such as Biotin-conjugated Bromodeoxyuridine (Bio-BrdU), the labeled DNA can be tracked by either radioactive signals or an antibody that recognizes Bio-BrdU, respectively. Normally, cycling cells, including cycling stem cells, can be labeled using this technique; but this labeling is lost during cell division. However, in some tissues, adult stem cells can retain the labeled DNA for a long period of time while the majority of the cycling progenitor cells lose the label during cell division. The LRC method has been used to assist in determining the putative stem cell locations in hair follicle, intestine, and bone marrow [5-8]. There are currently two mechanisms used to explain the LRC feature of adult stem cells. 1) The 'immortal DNA strand' model may explain why some, the most primitive, adult stem cells are able to retain labeled DNA strands for a long period of time, and 2) Adult stem cells in mammals either are maintained as quiescent cells or undergo very slow cycling and thus show long-term retaining of the labeled DNA. C Potten provided the first in vivo evidence to support an 'immortal DNA strand' mechanism that might play a role in protection of genome in intestinal stem cells. In this work, the old DNA strands in the stem cells were labeled during tissue regeneration using ${ }^{3} \mathrm{H}$-thymidine and the newly synthesized strands were labeled with a different marker (BrdU) to allow segregation of the two markers to be studied. He observed that the old strand label $\left({ }^{3} \mathrm{H}\right.$-thymidine $)$ was retained whereas the newly synthesized strands (BrdU) were lost following the second division of the stem cell [9]. Similarly, satellite cells assure postnatal skeletal muscle growth and repair, functioning as muscle stem cells. Using pulse-chase labeling with BrdU to mark the putative muscle stem cells, S Tajbakhsh identified a subpopulation of label-retaining satellite cells during growth and after injury. Strikingly, some of these cells displayed selective segregation of old DNA strand during mitosis in muscle fibers in vivo, indicating co-segregation of 'immortal' old DNA strands [10]. Studying fibroblast cell lines, J. Sherley found that some cultured cells also displayed the phenomenon of asymmetric segregation of DNA strand and this seemed to be regulated by the tumor suppressor p53, as loss of p53 resulted in impairment of this asymmetric segregation $[11,12]$.

A recent work published in Nature by Sean Morrison's group at the University of Michigan challenged the 'immortal DNA strand' model [13]. This group had previously reported that HSCs isolated using the Slam (signaling lymphocyte activation molecule) code (CD150+CD41-CD48-) 
were the most definitive HSCs [14]. First they determined how many bone marrow cells and HSCs are BrdU+ and found that BrdU labeling was not particularly sensitive or specific for HSCs; then they determined that the average rate of HSCs entering the cell cycle/day was $6 \%$. This information was used to develop a theoretical model of BrdU retention in the case of asymmetric vs. symmetric DNA segregation. They used two BrdU replacements, CldU and IdU, to label old DNA strands and newly synthesized DNA strands respectively. Using this strategy, they were able to test whether stem cells undergo asymmetric segregation of DNA strands. This mathematic model, based on either asymmetric segregation or random segregation of DNA strands during HSC division, predicts the percentage of CldU (or IdU) incorporated cells during the chasing period (around two months post injection), and the result supports the random segregation model. Furthermore, since less than $6 \%$ of Slam marked HSCs were BrdU-LRCs, the authors concluded that BrdU-LRCs are not sensitive enough to be used as HSC markers [13].

These provocative results provide strong evidence that these particular HSCs probably do not have an immortal DNA strand, and that on average they divide too frequently to retain BrdU over long periods. It may be premature, however, to conclude that no HSCs can ever have an immortal DNA strand, or to completely discard the concept that some HSCs can be LRC. In fact, BrdU positivity is always taken in context. For example in the Bmpr mutant animal model, expansion of trabecular bone results in HSC expansion, so LRCs were examined in association with bone and verified with other HSC markers (including cKit, Sca-1, and CD45). In this case, BrdU-LRC was used to assist in determining the putative HSC location, rather than as a sole HSC marker [8]. Instead, the work [13] by Morrison's group should provide a stimulus for further investigations, with particular attention being paid to the underlying assumptions on which their current conclusions rely: 1) the Slam (CD150+CD41-CD48-) marked HSCs represent the only and entire population of HSCs; and 2) all HSCs behave the same all of the time. Like all mathematical models, it is powerful but only to the extent that real HSCs adhere to the assumptions of the model.

If, however, these assumptions are not complete, the conclusions based on these assumptions may not hold. For example, it was demonstrated that in muscle stem cells only a very limited number of satellite cells showed selective retention of the labeled DNA strands [10], which may have failed to be predicted by the mathematical model used in the work by Kiel and coworkers [13]. Still, if 'immortal' DNA strands do exist in a rare population of HSCs, it remains the work of future investigations to prove this.

\section{References}

1 Reya T, Morrison SJ, Clarke MF, Weissman IL. Stem cells, cancer, and cancer stem cells. Nature 2001; 414:105-111.

2 Clarke MF, Dick JE, Dirks PB, et al. Cancer Stem Cells--Perspectives on Current Status and Future Directions: AACR Workshop on Cancer Stem Cells. Cancer research 2006; 66:9339-9344.

3 Wang JC, Dick JE. Cancer stem cells: lessons from leukemia. Trends in cell biology 2005; 15:494-501.

4 Cairns J. Mutation selection and the natural history of cancer. Nature 1975; 255:197-200.

5 Arai F, Hirao A, Ohmura M, et al. Tie2/angiopoietin-1 signaling regulates hematopoietic stem cell quiescence in the bone marrow niche. Cell 2004; 118:149-161.

6 Cotsarelis G, Sun TT, Lavker RM. Label-retaining cells reside in the bulge area of pilosebaceous unit: implications for follicular stem cells, hair cycle, and skin carcinogenesis. Cell 1990; 61:1329-1337.

7 Potten CS, Booth C, Pritchard DM. The intestinal epithelial stem cell: the mucosal governor. Int J Exp Pathol 1997; 78:219-243.

8 Zhang J, Niu C, Ye L, et al. Identification of the haematopoietic stem cell niche and control of the niche size. Nature 2003; 425:836-841.

9 Potten CS, Owen G, Booth D. Intestinal stem cells protect their genome by selective segregation of template DNA strands. Journal of cell science 2002; 115:2381-2388.

10 Shinin V, Gayraud-Morel B, Gomes D, Tajbakhsh S. Asymmetric division and cosegregation of template DNA strands in adult muscle satellite cells. Nat Cell Biol 2006; 8:677-687.

11 Merok JR, Lansita JA, Tunstead JR, Sherley JL. Cosegregation of chromosomes containing immortal DNA strands in cells that cycle with asymmetric stem cell kinetics. Cancer research 2002; 62:6791-6795.

12 Rambhatla L, Ram-Mohan S, Cheng JJ, Sherley JL. Immortal DNA strand cosegregation requires p53/IMPDH-dependent asymmetric self-renewal associated with adult stem cells. Cancer research 2005; 65:3155-3161.

13 Kiel MJ, He S, Ashkenazi R, et al. Haematopoietic stem cells do not asymmetrically segregate chromosomes or retain BrdU. Nature 2007; doi:10.1038/nature06115.

14 Kiel MJ, Yilmaz OH, Iwashita T, Yilmaz OH, Terhorst C, Morrison SJ. SLAM family receptors distinguish hematopoietic stem and progenitor cells and reveal endothelial niches for stem cells. Cell 2005; 121:1109-1121. 